\title{
Dynamic Model of Wind Speed Distribution in Wind Farm Considering the Impact of Wind Direction and Interference Effects
}

\author{
Zhe Dong ${ }^{1, a}$, Ran $\mathrm{Li}^{1, \mathrm{~b}}$ and Lei Dang ${ }^{1, \mathrm{c}}$ \\ ${ }^{1}$ Department of Electrical Engineering, North China Electric Power University, \\ Baoding, Hebei, 071000, China. \\ a199103088@qq.con, bhss0527@163.com , ${ }^{\mathrm{c}}$ danglei_mao@126.com
}

Keywords: Wind Speed Model, Interference Effect, Dynamic Distribution of Wind Speed.

\begin{abstract}
The accurate and practical wind speed model of wind farm is the basis of the relevant research work of wind power prediction. This paper based on the wind farm and the real-time data of wind turbine, put forward a kind of dynamic distribution model of wind speed that consider the wind turbine wake and wake flow superimposed effect and time delay effect, and considering the relative space position of wind machine in different wind direction. First analyzing the influence of the wake effect, wake flow superimposed effect, time delay effect and the change of wind direction on the wind speed in theory, establishing the mathematical model of wind speed that considered interference effects; secondly, based on fan location map and the measured wind speed of an actual wind farm, using the established the model to take simulation analysis of the distribution of wind speed, and taking deviation analysis on the simulation results and the actual wind speed data of the distribution dynamic model of wind speed and the traditional wind speed model put forward by this paper. The results show that the wind speed model put forward by this paper can more accurately simulated the attenuation, delay of the wind speed of the wind farm when the wind direction changed, and having practicability.
\end{abstract}

\section{Introduction}

Because the wind energy source having randomness and uncontrollability, the wind turbine is subjected to great disturbance almost every time, the disturbance making the output power of wind turbine power be fluctuated, resulting in that it is difficult to reach the ideal accuracy of power prediction, but also brought impact to the wind turbine and power system connected with it, such as, shortening the life of the wind machine, reducing the quality of power of power grid, and even brought voltage flicker problem[1-3]. Therefore, it is necessary to establish accurate wind speed model to simulate the changing fluctuation of wind speed.

\section{The Modeling}

Single Wind Wake Model. The wake of single wind turbine can be described by using the Jensen model [4]. The model thinks that the initial diameter of the cross section of the wake flow farm is wind wheel diameter, $r_{r o t}$ expressing the length of wind turbine blade. Using $d$ to express the distance of the downstream of turbine, $r_{w}$ expressing the radius of the cross-section of wake flow, then the radius of the cross-section of the wake flow can be expressed as:

$$
r_{w}=r_{r o t}+k * d
$$

in which $k$ being shape coefficient, usually the onshore wind farm taking $k=0.075$, offshore wind farm being $k=0.04$ [5].Using $v_{0}, v_{w 0}, v_{w}$ respectively to expresses the natural wind speed, the wind speed of the back of wind turbine, the speed of wake flow. We can get the wind speed of the back of wind turbine by momentum theory: 


$$
v_{w o}=(1-a) \mathrm{v}_{0}
$$

$a$ is the induction factor of axial, $a=1-\sqrt{1-C_{T}}, C_{T}$ is thrust coefficient of wind turbine.

The speed of the wind turbine in the wake:

$$
v_{w}(d)=v_{0}\left[1-a\left(\frac{r_{r o t}}{r_{w}}\right)^{2}\right]
$$

The Model of Overlay of Wake Flow. For two fans with the relative distance that being along wind direction, the length of the fan blade of downstream being $r_{r o t}$, swept area being $A_{r o t}$. When the wake flow of a fan of the upstream reached $d$, the wake radius being $r_{w}(\mathrm{~d})$, the effect area of the wake flow being $A_{w}(\mathrm{~d})$. Regulating the overlap area of the other two units is $A_{s}$, according to the degree of overlap, we can divide the influence of the wake flow of upstream fan on the fan of downstream into three kinds: under lap, completing overlap, partial overlap. The overlap area of the former two cases are $0, A_{\text {rot }}$. For partial overlap, according to the overlapping area it can be divided into two cases in figure 1, and respectively giving the calculation method of overlapping area.

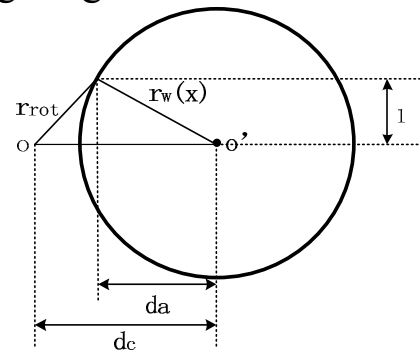

(a) $r_{w}(\mathrm{~d})<d_{c}<r_{w}(\mathrm{~d})+r_{r o t}$

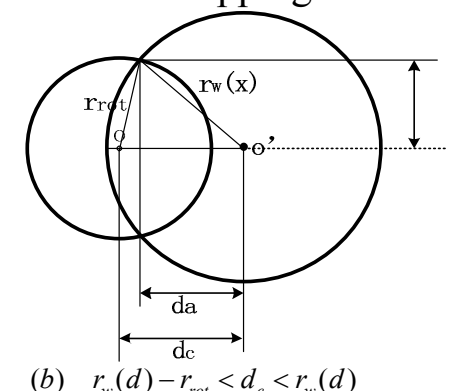

(b) $r_{w}(d)-r_{r o t}<d_{c}<r_{w}(d)$

Figure 1 Schematic diagram of overlap of fan

$$
\text { Case (a) } \quad A_{s}=r_{r o t}{ }^{2} \arccos \left(\frac{d_{c}-d_{a}}{r_{r o t}}\right)+r_{w}(d)^{2} \arccos \left(\frac{d_{a}}{r_{w}(d)}\right)-d_{c} \cdot l
$$

Case (b) $A_{s}=r_{\text {rot }}{ }^{2} \arccos \frac{r_{r o t}{ }^{2}+d_{c}{ }^{2}-r_{w}(d)^{2}}{2 d_{c} r_{r o t}}+r_{w}(d)^{2} \arccos \frac{r_{w}(d)^{2}+d_{c}{ }^{2}-r_{r o t}{ }^{2}}{2 d_{c} r_{w}(d)}-d_{c} l$

According to the law of conservation of momentum, we can calculate the input speed of any fan $i$ in the wind farm by formula (6):

$$
v_{i}=\sqrt{v_{i 0}^{2}-\sum_{\substack{k=1 \\ k \neq i}}^{n} \beta_{i k}\left[v_{i 0}^{2}-v_{w-i k}\left(d_{i k}\right)^{2}\right]}
$$

in the formula, $v_{i}$ expressing the wind speed effecting on the fan $i ; v_{i 0}$ expressing the wind speed the $i$ fan without the effect of any wake flow and tower shadow; $\beta_{i k}$ being the ratio of the projection area of the $k$ fan in the wind wheel of the $i$ fan compared with the area of wind wheel of the $i$ fan: $\beta_{i k}=A_{s-i k} / A_{r o t-i} ; v_{w-i k}\left(d_{i k}\right)$ being the wind speed of wake flow when the $k$ fan effecting on the $i$ fan; $d_{i k}$ expressing the relative distance downwind between fan $i$ and fan $k$.

Time Delay Model of The Wake Flow of Fan. Considering the time delay of wind speed between fans, thinking approximately that the wind direction of transmission being the current wind direction, the route of transmission being a straight line, the transmission speed being the wind speed of the back of the upstream fan, ignoring the loss of wind speed and the abouchement of natural wind speed in the transmission process. We can calculate and get the capturing speed $v_{i}(\mathrm{t})$ of unit $i$ at the $\mathrm{t}$ moment: 


$$
v_{i}(t)=\sqrt{v_{i 0}(t)^{2}-\sum_{\substack{k=1 \\ k \neq i}}^{n} \beta_{i k}\left[v_{i 0}^{2}(t)-v_{w-i k}{ }^{2}\left(d_{i k}, t-T_{i k}\right)\right]}
$$

in the formula, $T_{i k}=v_{w 0-k} / d_{i k}$ said the time delay effect of the wake flow of unit $k$ to unit $i$.

The Distribution Model of Dynamic Space of Fan. In the actual operation of wind farm, wind direction is dynamic. We must be based on the distribution model of dynamic space of fan that considering the effect of the change of wind direction on the wind flow.

(1)The establishment of the initial coordinate system

Taking the counter-clockwise direction as the forward direction, the fan in the north side of the wind farm is the pole, taking east direction as the polar axis. According to the distribution of the fan position, to obtain the initial coordinate of $n$ fan in the wind farm. The coordinate of any fan $i$ in the wind farm is $\rho_{i}\left(r_{i}, \theta_{i}\right), r_{i}, \theta_{i}$ respectively said the distance between unit $i$ and pole, the included angle between the unit $i$ and the polar axis.

(2) The transformation of dynamic coordinate

Taking the north direction as the wind direction angle of 0 degree, clockwise direction is positive, the wind direction angle at any time being $\beta$. According the wind direction angle to take rotation transformation on the initial coordinate, taking clockwise rotation angle $\beta$ on the pole axis of new coordinate to get the pole axis of the initial coordinate, after transformation, the coordinate of any fan $i$ in the wind farm is $\rho_{i}^{\prime}\left(r_{i}^{\prime}, \theta_{i}^{\prime}\right)$, which $r_{i}^{\prime}=r_{i}, \theta_{i}^{\prime}=\theta+\beta$.

(3) Calculating the relative position of fan according to the location of new coordinate

The new coordinate after transformation can be decomposed to obtain the position of each unit in downwind and crosswind directions. In which, the coordinate of any fan $i$ is $\rho_{i}^{\prime}\left(r_{i}^{\prime}, \theta_{i}^{\prime}\right)$, the position of it in the downwind and crosswind direction respectively being $d_{s i}=r_{i}{ }^{\prime} \sin \theta^{\prime}, d_{c i}=r_{i}{ }^{\prime} \cos \theta^{\prime}$. The downwind and crosswind directions of all $n$ fans in the wind farm are:

$$
D_{S}=\left[d_{s 1}, d_{s 2}, \ldots, d_{s n}\right], D_{c}=\left[d_{c 1}, d_{c 2}, \ldots, d_{c n}\right]
$$

Sorting the fans in the wind farm from small to large according to the wind direction, we can get the collection of all the $k$ fans of the upstream of any unit $i$, and can calculate the relative position of the downwind and crosswind of the relative unit $i$ of the $k$ fans. Assuming that the number of upstream unit as the $1,2, \ldots k$. The relative position of the downwind and crosswind of the relative unit $i$ of the fan $k$ respectively are :

$$
\begin{aligned}
& D_{s i}=\left[d_{s i 1}, d_{s i 2}, \ldots, d_{s i k}\right]=\left[d_{s 1}-d_{s i}, d_{s 2}-d_{s i}, \ldots, d_{s k}-d_{s i}\right] \\
& D_{c i}=\left[d_{c i 1}, d_{c i 2}, \ldots, d_{c i k}\right]=\left[d_{c 1}-d_{c i}, d_{c 2}-d_{c i}, \ldots, d_{c k}-d_{c i}\right]
\end{aligned}
$$

The establishment of the dynamic wind speed distribution model of wind farm. The distribution curve of the model of this paper using the location of wind farm is according to the method of setting model of the above analysis to set the initial space model, taking the real time data of anemometer tower as the input of model. According to the wind direction to take the coordinate transformation, calculating the relative space position of the unit in the current moment, determining the marshalling sequence of units of upstream and downstream and the relative distance of crosswind, then calculating the interaction of the wake flow of the units. At the same time, through the relative distance of the downwind of units and the wind speed of the back of the units to determine the travel time of the wake flow, calculating the wake effects of time delay. Finally, the model gives the real time speed of each fan in wind farm as result. 


\section{Examples}

This paper takes a wind farm as the research object for the wind speed modeling and verification, the electric field being equipped with 33 sets of $1.5 \mathrm{MW}$ wind turbines, blade length being $37.5 \mathrm{~m}$. Figure 2 shows the location map of part of the fan used in the simulation of the section.

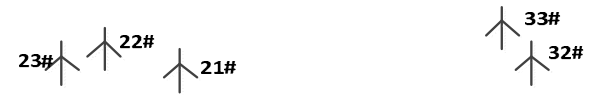

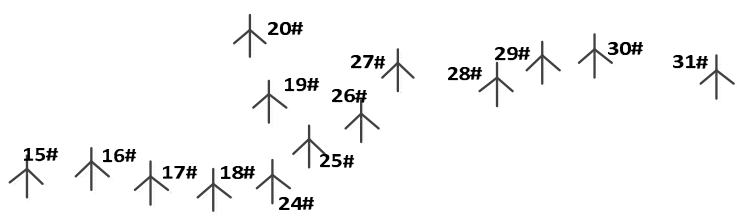

Figure 2 The location map of partial fans of wind farm

This section took simulation comparison on the traditional distribution model of wind speed and the distribution model of dynamic wind speed put forward by this paper that considering the wind direction and interference effect of fan. The selected wind direction of the traditional distribution model of wind speed is constant and is the main wind direction of the current season: north wind ( 0 degrees); the distribution model of dynamic wind speed considering wind direction and interference effect of fan took the real time data of anemometer tower as input.
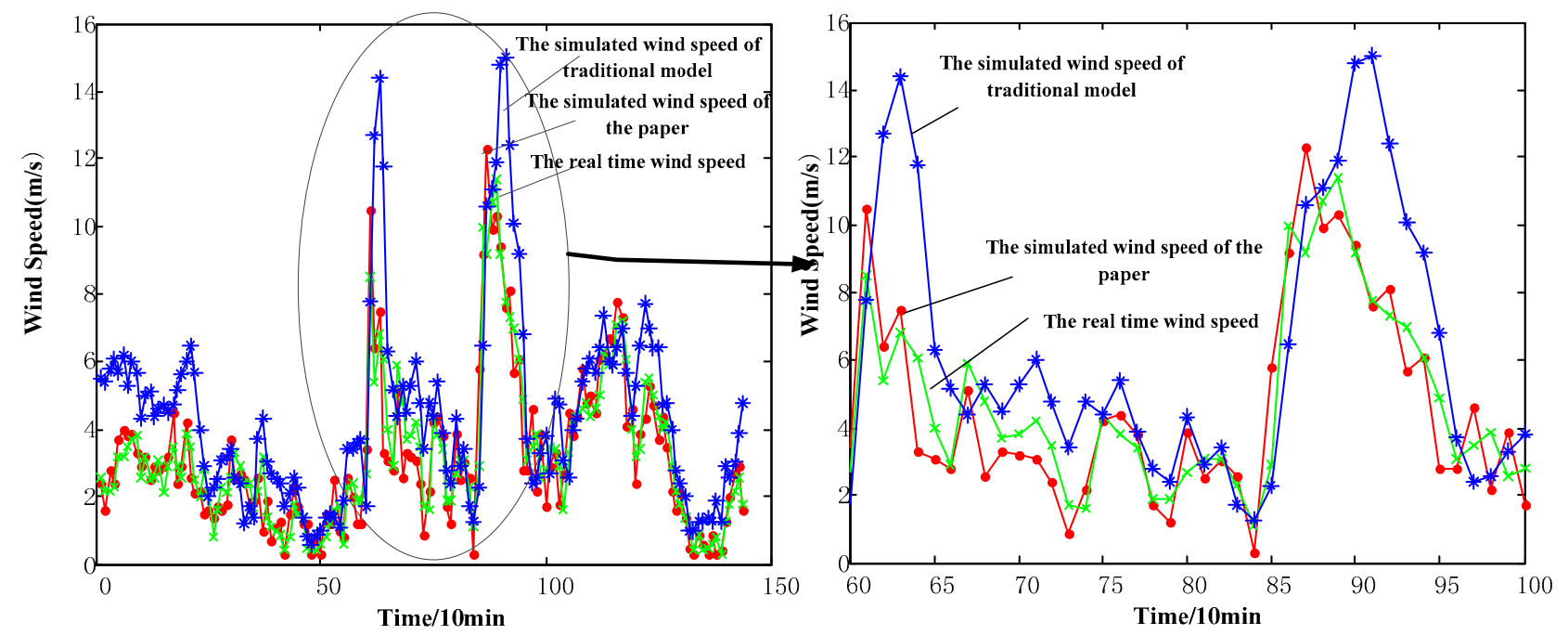

Figure 3 The contrast of the simulated wind speed and the real time wind speed of the fan

Extracting the wind speed of WT27 from the output results of two kinds model, comparing the actual wind speed of the fan, the result being shown in figure 3. The distribution model of dynamic wind speed considering the effect of wind direction got that the simulated wind wave is more consistent with the real time wind speed wave, the amplitude being basically identical. Figure 4 and table 1 took deviation analysis on the simulated wind speed and the real time wind speed of the two models. We can see that the deviation between the simulated wind speed and the real time wind speed of the traditional model is basically negative, namely the actual wind speed is less than the simulated wind speed. This is because the traditional model in the simulation took the north wind and made it be unchangeable, and then the location of WT27 is basically not affected by effects of upstream wake flow of fan; and when the wind in the northwest and northeast slightly offset, while the offset angle is small, the fan in this location being too close, the slightly change of the relative position of the fan WT27 that can make the fan WT27 be in the influence range of wake flow of WT26 or WT28, 
resulting in the reduce of wind speed of fan WT27, showing as that the actual wind speed is smaller than the simulated wind speed. And for the distribution model of dynamic wind speed of the wind farm put forward in this paper, because considering the influence of the wind speed change on the relative position of the fan, the deviation being on the fluctuation of the $0 \mathrm{~m} / \mathrm{s}$, the deviation of model was obviously smaller than the deviation of traditional model. Table 1 gave the data of deviation of two kinds of models, the accuracy of the distribution model of dynamic wind speed considering the influence of wind direction got greatly improved compared with the traditional model of the wind speed, can be more accurately to describe the dynamic changes of wind speed of wind farm.

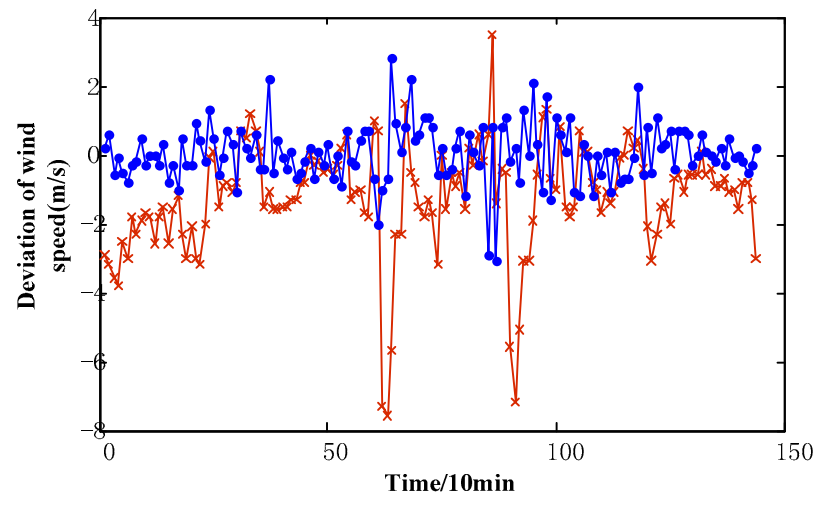

Figure 4 The deviation analysis of the real time wind speed and the simulated wind speed

Table 1 Comparison of the deviation of simulated wind speed of the two kinds of model

\begin{tabular}{cccc}
\hline & $\begin{array}{c}\text { The biggest deviation } \\
(\mathrm{m} / \mathrm{s})\end{array}$ & $\begin{array}{c}\text { The smallest deviation } \\
(\mathrm{m} / \mathrm{s})\end{array}$ & $\begin{array}{c}\text { The average deviation } \\
(\mathrm{m} / \mathrm{s})\end{array}$ \\
Traditional model & 7.6 & 0 & 1.4917 \\
The model of this paper & 2.9 & 0 & 0.6201 \\
\hline
\end{tabular}

\section{References}

[1] Sun Tao, Wang Weisheng, Dai Huizhu, et: Power System Technology, in Chinese, Vol.27(2003),p.62-66+70.

[2] Ma Xinxia, Song Mingzhong, Li Yongguang: Journal of Shanghai University of Electric Power, in Chinese, Vol.22(2006),p.283-286+291.

[3] Ceng Lihua, Wang Feng, Liu Deyou: Proceedings of the CSEE, in Chinese, Vol.31(2011),p.37-42.

[4] Cao Na, Zhao Haixiang, Ren Puchun, et: Proceedings of the CSEE, in Chinese, Vol.27(2007),p.68-72.

[5] Rathmann O, Barthelmie R J, Frandsen S: Turbine wake model forwind resource software (European Wind Energy Conference and Exhibition, Athens, 2006) . 